\title{
Mitochondrial Energy Production Correlates With the Age-Related BMI
}

\author{
SASKIA B. WORTMANN, HEIDI ZWEERS-VAN ESSEN, RICHARD J. T. RODENBURG, LAMBERT P. VAN DEN HEUVEL, \\ MAAIKE C. DE VRIES, ELLEN RASMUSSEN-CONRAD, JAN A. M. SMEITINK, AND EVA MORAVA \\ Department of Pediatrics [S.B.W., M.C.V., J.A.M.S., E.M.], Department of Dietetics [H.Z.E., E.R.-C.], Laboratory of Pediatric and \\ Neurology [R.J.T.R., L.P.H.], Radboud University Medical Center Nijmegen, 6500 HB Nijmegen, The Netherlands
}

\begin{abstract}
Besides characteristic neurologic and musculoskeletal symptoms, children with mitochondrial dysfunction often present with feeding problems and failure to thrive. Substrate depletion for the respiratory chain has an effect on energy expenditure. Secondary mitochondrial dysfunction has been reported in severe chronic malnutrition. We evaluated the nutritional state, the growth parameters, and the metabolic condition in 172 children undergoing muscle biopsy for a suspected disorder of the oxidative phosphorylation system (OXPHOS). We performed dietary evaluation and initiated nutritional intervention when needed before the biopsy. Mitochondrial dysfunction was confirmed by detection of enzyme-complex deficiencies and/or by mutations in 83 children, in 14 patients no biochemical abnormalities were found. In the whole study group, and in the subgroup with enzyme-complex deficiency and/or mutation, a significant correlation was found between the mitochondrial production of adenosine triphosphate (ATP) and the age-related body mass index (BMI). Nutritional state and growth should be considered by interpreting the results of ATP-production in fresh muscle biopsy. Because of a positive correlation between the age-appropriate BMI and the ATP-production, we strongly advise optimizing the nutritional state preceding the muscle biopsy in children with a suspected OXPHOS-disorder. Dietary intervention remains although challenging because of frequent gastrointestinal problems and eating disorders. (Pediatr Res 65: 103-108, 2009)
\end{abstract}

Is: decreased mitochondrial energy production a consequence of severe respiratory chain dysfunction itself, or is it influenced by the actual nutritional state of the child with a severe chronic disease? This question appears frequently in the clinical practice, especially by the interpretation of the results of respiratory chain enzyme measurements in muscle. Secondary deterioration of mitochondrial function has been reported in patients with different chronic disorders, anorexia, and cancerrelated malnutrition (1-4). Therefore, one should consider that some children with severe malnutrition have only a secondary mitochondrial disease because of chronic substrate depletion $(5,6)$. Chronic vitamin deficiency affects the mitochondrial function as well (7-9). Previously, we measured an increased mitochondrial production of adenosine triphosphate (ATP) in a few children with a second biopsy after the improvement of the nutritional condition (5). Secondary mi-

Received April 17, 2008; accepted August 7, 2008.

Correspondence: Eva Morava, M.D., Ph.D., Department of Pediatrics, Nijmegen Center for Mitochondrial Disorders, Radboud University Nijmegen Medical Center, Nijmegen, P.O. Box 9101, 6500 HB Nijmegen, The Netherlands; e-mail: E.Morava@cukz.umcn.nl tochondrial dysfunction was also found in several patients with variable inborn errors and insufficient nutrition $(6,10)$. We assume that nutritional intervention with an individualized diet and optimal dietary intake in children with a mitochondrial disease improves the energy generating capacity, the clinical condition, and the quality of life in patients.

We hypothesized a significant correlation between the growth and nutritional state and the energy production in children with both primary and secondary dysfunction of the oxidative phosphorylation system (OXPHOS). To test our hypothesis, we designed a prospective investigation to evaluate a possible correlation between the growth parameters, nutritional state, metabolic condition, and the OXPHOS function in patients with a suspected mitochondrial disease. Children with the clinical diagnosis of mitochondrial dysfunction based on the mitochondrial disease criteria (MDC; 10) were consecutively included in the evaluation. The body mass, muscle mass, and the nutritional state play an important role for the mitochondrial energy production capacity $(1,2)$. As part of the standard work-up, before performing a muscle biopsy for a suspected mitochondrial dysfunction, we evaluate the growth parameters and perform nutritional evaluation in children with a decreased body weight for age or a decline in growth or thrive before the first outpatient visit. All children received dietary advice for nutritional intervention and a follow-up consult in the consecutive work-up period.

\section{METHODS}

We prospectively evaluated the data of 172 children based on a MDC score $\geq 3$ [score 2-4 suggests a "possible mitochondrial disorder", 5-7 is comparable with a "probable mitochondrial disorder"; score 8-12 confirms the diagnosis "definite mitochondrial disorder" (10)] who underwent a muscle biopsy for a suspected mitochondrial disease in the period of 2005-2007. The study has been carried out in the Netherlands in accordance with the applicable rules concerning the review of research ethics committees (Commissie Mensgebonden Onderzoek Regio Arnhem-Nijmegen) and informed consent. The MDC score was calculated based on clinical symptoms, metabolic alterations, and abnormal neuroimaging features. Initially, after the first outpatient visit, as part of the routine work-up for a suspected disorder in the OXPHOS, all children underwent a standard diagnostic protocol of multiple investigations, including anthropometric measurements, Electrocardiogram (ECG), chest $\mathrm{x}$-ray, electroencephalography (EEG), brain auditory evoked potential (BAEP), visual evoked potential (VEP), sensory evoked potentials (SEP), and a cranial magnetic resonance imaging (MRI) before the muscle biopsy. Serum lactic acid (multiple measurements), pyruvic acid levels, blood

Abbreviations: ATP, adenosine triphosphate; MDC, mitochondrial disease criteria; OXPHOS, oxidative phosphorylation 
gas, serum acyl-carnitine, amino acid, and urine organic acid profiles were analyzed in all children. Cerebral spinal fluid (CSF) investigations were not performed in all patients.

The surgical muscle and skin biopsy was performed under general anesthesia in all children. Fibroblasts were cultured and the activity of pyruvate dehydrogenase complex (PDHc) and the respiratory enzyme-complexes I-V were measured according to standard protocols. Parallel with routine immunehistologic and (in case of abnormal histology, positive family history, muscle cramps, increase creatin kinase or proven mutation) upon electronmicroscopic analysis, the ATP-production from pyruvate oxidation and the activity of PDHc and the respiratory enzyme-complexes I-V were measured in the fresh muscle sample according to the methods described previously $(11,12)$. Based on the histologic findings, appropriate genetic studies were initiated. Depending on the expression of mitochondrial function in fibroblasts and the existence of isolated or complex respiratory enzyme abnormalities mtDNA sequence analysis, mutation analysis of the POLG1 gene (MIM *174763) and sequence analysis of the structural genes of the individual complexes were performed in all cases.

One hundred seventy two children with the clinical diagnosis of mitochondrial dysfunction based on the MDC score were consecutively included in the evaluation. Before performing a muscle biopsy for a suspected mitochondrial dysfunction, we performed detailed nutritional evaluation in children with a significantly decreased body weight for age or a significant decline in growth or thrive in the last 6 mo period before the first outpatient visit. Additional evaluation of cell count, cell volumes, $\mathrm{Hb}$ concentration, albumin, triglycerides, cholesterol, uric acid, minerals, alkaline phosphate, vitamin B12, and folic acid levels were initiated. All children received dietary advice for nutritional intervention for at least a 3-mo period. Dietary advice included the correction of the caloric intake according to age appropriate needs, supplementation of vitamins and minerals, if insufficient, the use of complex carbohydrates instead of simple sugars and optimizing the daily eating structure.

Height and weight were measured and evaluated using Growth Analyser (Growth analyser 3.5, stichting kind en groei) at least twice in all children. If indicated growth charts according to the ethnic background were used. All data were analyzed using SPSS 14.0. Anthropometric data and ATPproduction are normally distributed in our population; therefore, we used the Pearsons correlation to evaluate a possible statistical significant correlation.

\section{RESULTS}

Data from 172 children (107 boys, 65 girls) were obtained. The age at biopsy ranged from 2 mo up to 19 y (mean age $5.04 \mathrm{y}, \mathrm{SD} 3.48$ ). The height for age varied between $-5.88 \mathrm{SD}$ and $+2.66 \mathrm{SD}$, the weight for age between $-6.71 \mathrm{SD}$ and $+4.11 \mathrm{SD}$. The body mass index (BMI) was calculated and ranged between 11 and $29.5 \mathrm{~kg} / \mathrm{m}^{2}$ (-5.1 SD up to +3.48 SD). In 26 patients, a pathologic mutation was confirmed, 12 in the mitochondrial DNA and 14 in the nuclear DNA (Table 1). All but three children with a confirmed genetic etiology demonstrated a decreased OXPHOS enzyme activity; all but one of these children had a decreased ATP-production in muscle. In total, a deficiency of one or more enzyme complexes of the OXPHOS-system was found in 80 patients. Seventy five patients had a decreased ATP-production from pyruvate oxidation without enzyme deficiency or mutation. In 20 children, ATP-production from pyruvate oxidation in muscle was within the normal range, although six of these children showed an enzyme deficiency and one harbored a PDHAl (MIM *300502) mutation. In summary, in 14 children, no biochemical abnormality was found.

The data of the correlation analysis for the whole group $(n=172)$ are shown in Table 2; a and Figure 1. There was a significant correlation between the parameters ATPproduction rate from pyruvate oxidation versus weight for age and BMI for age, respectively. No correlation was found for the length (height) of the patient group.
We additionally defined and analyzed three subgroups with proven mitochondrial disorder within the whole study group $(n=172)$; proven either by a pathogenic mutation (group 1; $n=26$ ), a deficiency of one or more enzyme-complexes of the OXPHOS system (group $2 ; n=80$ ), and a third group with the combination of these two diagnostic criteria (group 3; $n=83$ ). For two of these subgroups (group 2 and 3), there is a correlation between the ATP-production and the BMI for age (see Table 2; b-d; and for group 3 Fig. 2). No correlation was found for the ATP-production versus the age appropriate BMI in the small group of patients with proven mutation (group 1), most likely because of the small group size $(n=26)$. There was no correlation between the weight for age, height for age, and the ATP-production for either group (group 1, 2, and 3). In summary, the patients with a clinically suspected and enzymatically (or by mutation analysis) proven mitochondrial disease showed a correlation between their muscle ATPproduction and their age-related BMI, but not for the other anthropometric parameters.

Furthermore, we analyzed the correlation of the growth parameters in children showing any signs of OXPHOS dysfunction in muscle (group $5 ; n=158$ ) including OXPHOS complex deficiencies and proven mutations (group 3;n= 83 ) and all children with an isolated ATP deficiency (group $4 ; n=75$ )

Based on this criteria, this group 4 encompassing 75 children with a decreased ATP-production without a proven mutation or enzyme-complex deficiency would include patients with a further not defined mitochondrial dysfunction and the patients with secondary mitochondrial dysfunction. In this group, we did not see any correlation between the anthropometrical data and the ATP-production (Table 2; e).

In the group including all children with any signs of OXPHOS dysfunction in muscle (group 5), we saw a significant correlation between BMI for age and the ATP-production (Table 2f) as we also observed in group 3 (Table 2d, Fig. 2).

Detailed immune-histologic and, if indicated, electron microscopic analysis was performed. In the subgroup 1 with proven mutation, 20 of 26 patients showed abnormalities (Table 1), including fiber type dysproportion $(n=20)$, ragged red fibers $(n=2)$, COX-deficiency $(n=3)$, multicores $(n=$ $1)$, and abnormal mitochondria on electron microscopy $(n=$ 5). For details concerning the other subgroups, see Table 3.

The nutritional state of all children was at least once evaluated in detail by our dietician. Children with severe feeding problems or failure to thrive got a personalized, age-appropriate feeding scheme. Concerning the whole group 37 of all 172 children (22\%) got tube-feeding at the time of muscle biopsy, from the group with proven mutation 11 of 26 $(42 \%)$ needed this aggressive treatment.

The nutritional state of 35 children with a weight $\leq-2.5$ SD for age, or decreasing growth curve was evaluated in detail. Insufficient caloric intake was confirmed in 18 cases. Insufficient vitamin or mineral intake was found in three children. Eating disorders were confirmed in five cases. Abnormal eating habits and unstructured intake was found in four patients. Tube feeding was indicated in 13 cases. In this described subgroup, all but one child had a decreased ATP- 


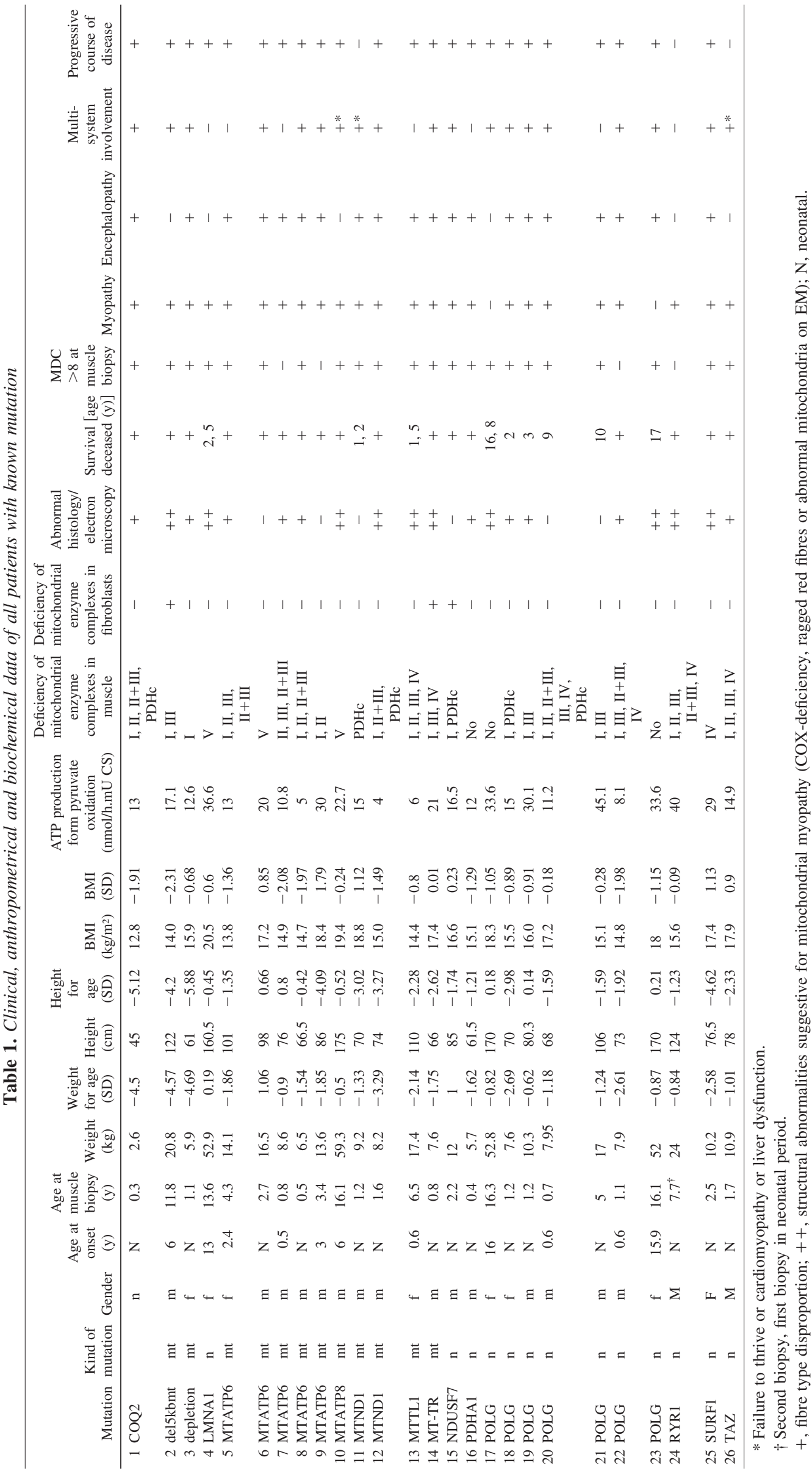


Table 2. Pearson correlation and p score of anthropometric data vs. ATP-production

\begin{tabular}{|c|c|c|c|c|}
\hline Group & & $\begin{array}{l}\text { ATP } v s . \text { height } \\
\text { for age }\end{array}$ & $\begin{array}{l}\text { ATP vs. weight } \\
\text { for age }\end{array}$ & $\begin{array}{l}\text { ATP vs. BMI } \\
\text { for age }\end{array}$ \\
\hline \multicolumn{5}{|l|}{ Whole group $(N=172)$} \\
\hline & Correlation & 0.149 & $0.216^{*}$ & $0.238^{*}$ \\
\hline \multicolumn{5}{|c|}{ Group 1 (proven mutation, $N=26$ ) } \\
\hline & Correlation & 0.228 & 0.370 & 0.360 \\
\hline & $p$ & 0.262 & 0.063 & 0.071 \\
\hline & Correlation & 0.048 & 0.200 & $0.254 \dagger$ \\
\hline & $p$ & 0.676 & 0.075 & 0.023 \\
\hline \multicolumn{5}{|c|}{$\begin{array}{l}\text { Group } 3 \text { (deficiency of one or more mitochondrial } \\
\text { enzymecomplexes and/or proven mutation, } N=83 \text { ) }\end{array}$} \\
\hline & Correlation & 0.069 & 0.206 & $0.247 \dagger$ \\
\hline & $p$ & 0.533 & 0.062 & 0.025 \\
\hline & Correlation & 0.068 & 0.145 & $0.209^{*}$ \\
\hline & $p$ & 0.396 & 0.068 & 0.009 \\
\hline
\end{tabular}

* Correlation is significant at the 0.01 level (2-tailed).
† Correlation is significant at the 0.05 level (2-tailed).

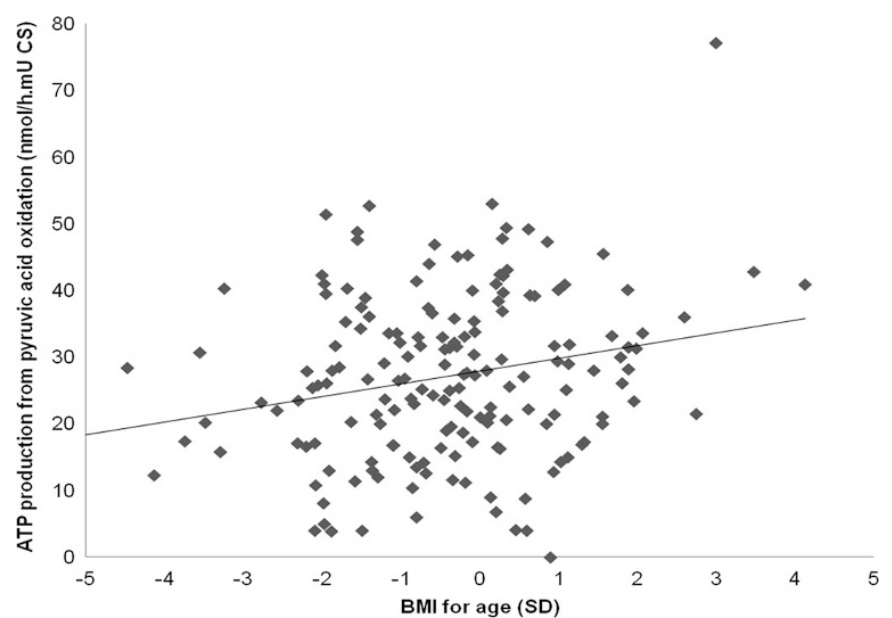

Figure 1. Age-related BMI (in SD) versus ATP-production from pyruvic acid oxidation (in $\mathrm{nmol} / \mathrm{h}$. mU CS) for the whole group $(n=172)$. Pearsons correlation 0.238 , which is significant at the 0.01 level (two-tailed).

production; a high percentage of children harbored a mutation $(8 / 35=23 \%)$ or had a deficiency of one or more mitochondrial enzyme complexes $(21 / 35=60 \%)$. In 19 of these 35 patients with very poor growth parameters alanine in serum, as a parameter of long time ineffective OXPHOS was measured at time of the biopsy. In three of these patients it was elevated, the other children had normal alanine-levels. One patient had a mild anemia (hemoglobine $6.6 \mathrm{mM}$ ), no other abnormalities were found in the screening laboratory.

Blood cell count, minerals, liver, and kidney function tests, albumine, cholesterol and triglycerids, blood gas, glucose, and uric acid in blood were normal in nearly all 172 children. Evaluating the alanine and lactate levels was not part of the prospective set up; some children underwent alanine and lactate testing not at the time of muscle biopsy, but some time in advance. Out of the 101 children in whom

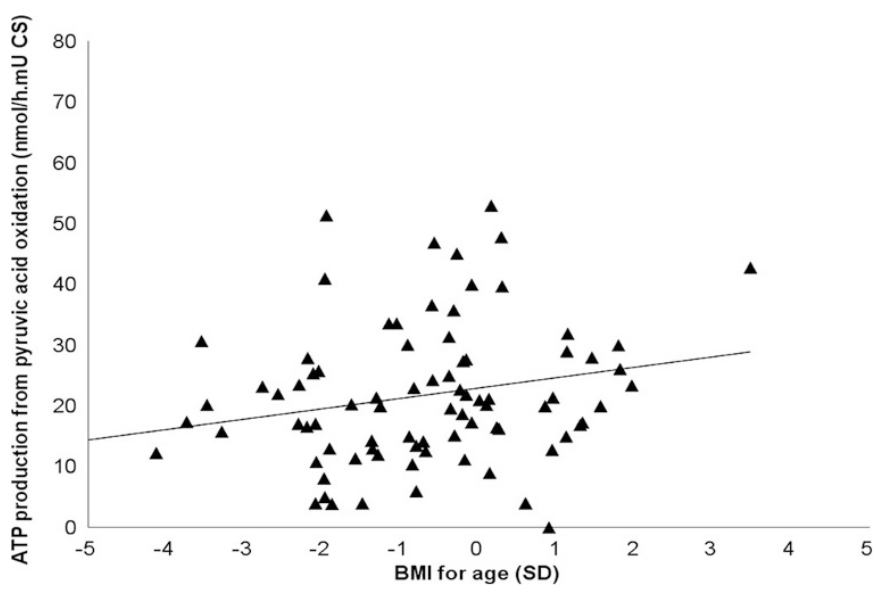

Figure 2. Age-related BMI (in SD) versus ATP-production from pyruvic acid oxidation (in $\mathrm{nmol} / \mathrm{h}$. mU CS) for the patients with deficiency of one or more mitochondrial enzyme-complexes and/or proven mutation (subgroup 3; $n=83$ ). Pearsons correlation 0.247, which is significant at the 0.05 level (two-tailed).

alanine was evaluated at time of the biopsy, 30 had elevated levels. Lactic acid had been tested in 137 children and 75 of them had increased blood levels. No functional B12 deficiency was detected in the group of children with failure to thrive. No biochemical signs of thiamine deficiency were confirmed.

For the clinical data of the patients, see Table 3.

\section{DISCUSSION}

Children with a suspected mitochondrial disorder often suffer from feeding problems and failure to thrive. Before a muscle biopsy, the question raises whether decreased mitochondrial energy production is a consequence of severe respiratory chain dysfunction itself, or if it is influenced by the 
Table 3. Clinical and biochemical patient characteristics

\begin{tabular}{|c|c|c|c|c|c|c|}
\hline & $\begin{array}{l}\text { Group 1 } \\
(N=26)\end{array}$ & $\begin{array}{r}\text { Group 2 } \\
(N=80)\end{array}$ & $\begin{array}{l}\text { Group } 3 \\
(N=83)\end{array}$ & $\begin{array}{l}\text { Group } 4 \\
(N=75)\end{array}$ & $\begin{array}{r}\text { Group 5 } \\
(N=158)\end{array}$ & $\begin{array}{l}\text { Whole group } \\
(N=172)\end{array}$ \\
\hline Myopathy & 24 & 15 & 39 & 25 & 64 & 70 \\
\hline Encephalomyopathy & 20 & 27 & 47 & 39 & 86 & 90 \\
\hline Leigh-like syndrome & 2 & 4 & 6 & 3 & 9 & 9 \\
\hline Severe visual loss & 1 & 4 & 5 & 5 & 10 & 10 \\
\hline Sensory neural hearing loss & 1 & 2 & 3 & 1 & 4 & 4 \\
\hline Dystonia & 1 & 0 & 1 & 1 & 2 & 2 \\
\hline Multi-system involvement & 19 & 12 & 31 & 11 & 42 & 43 \\
\hline Renal tubular acidosis & 0 & 0 & 0 & 1 & 1 & 1 \\
\hline Cardiomyopathy & 2 & 0 & 2 & 0 & 2 & 2 \\
\hline Liver dysfunction & 6 & 3 & 9 & 5 & 14 & 15 \\
\hline $\begin{array}{l}\text { Severe malabsorption/severely impaired bowel } \\
\text { mobility }\end{array}$ & 0 & 4 & 4 & 2 & 6 & 6 \\
\hline $\begin{array}{l}\text { Organic aciduria (3-MGA, EMA or } \\
\text { alpha-ketoglutaric aciduria) }\end{array}$ & 4 & 2 & 6 & 2 & 8 & 8 \\
\hline $\begin{array}{l}\text { Abnormal histology or electron- microscopy } \\
\text { in muscle biopsy }\end{array}$ & 20 & 2 & 22 & 3 & 25 & 25 \\
\hline $\begin{array}{l}\text { Deficiency of mitochondrial enzyme } \\
\text { complex(es) in cultured fibroblasts }\end{array}$ & 3 & 4 & 7 & 0 & 7 & 7 \\
\hline
\end{tabular}

Group 1, proven mutation; Group 2, deficiency of one or more mitochondrial enzyme-complexes; Group 3, deficiency of one or more mitochondrial

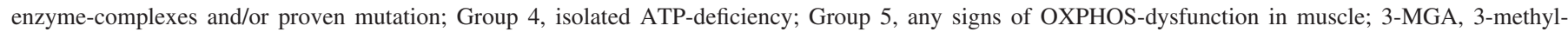
glutaconic aciduria; EMA, ethylmalonic aciduria.

actual nutritional state of the child with a severe chronic disease. We investigated the correlation between the growth parameters and the ATP-production in fresh muscle biopsy in 172 children with a suspected OXPHOS disorder. From our study, we conclude that a higher age-related BMI, thus a possible better nutritional state is associated with a higher ATP production, suggesting a higher mitochondrial energygenerating capacity.

One would expect that obese children would show a decreasing trend in ATP-production, for example, because of physical inactivity. It is impossible to conclude this from our data, as only six children met the definition of obesity with a BMI $\geq 2$ SD. From these children, four had a decreased ATP-production, none harbored a mutation, and one had an enzyme deficiency. What we can conclude is that obesity was nearly seen in the population of children with a suspected or proven mitochondrial disorder.

To prevent possible secondary mitochondrial abnormalities, we offered nutritional intervention for our patients. Although we confirmed a significant correlation between weight for age and age-related BMI in the prospectively evaluated unbiased study group $(n=172)$, and for the age appropriate BMI in the group with enzyme deficiencies $(n=80)$, we could not confirm a correlation between growth parameters and energy production in the small patient group with proven mutations $(n=26$, for details see Table 1$)$.

This might be biased because of the small group size, but these findings underline our earlier results in a comparable small group of 10 children undergoing a second muscle biopsy for different reasons after dietary advice and nutritional intervention (5). In seven children, an age-appropriate diet could be achieved, in four children acquiring supplementary or total tube feeding. In six of these children ATP-production dramatically improved, in some nearly doubled, with four of them achieving the normal range, afterwards.
The ATP-production in the three children with persistent poor nutritional state remained the same; interestingly, these also were the children with a deficiency of respiratory chain enzyme complexes. Because the nutritional component was optimized in these patients, we concluded that the genetic factor appears to be the primary component in influencing the growth parameters with proven primary mitochondrial disease.

Concerning our recent data it seems that in the bigger group of children with a mitochondrial disorder, a positive effect on the energy expenditure is seen when the nutritional state improves. For the group of all children showing any signs of OXPHOS dysfunction in muscle (group 5, Table 2f), a significant correlation between the age-related BMI and ATPproduction is seen. One might argue that this group also encompasses children with secondary mitochondrial dysfunction because of other underlying diseases. This is right, some of these children will suffer from a so far undefined mitochondrial dysfunction; partially because of a primary mitochondrial disease, but probably, in other cases secondary to another neurologic, genetic, syndromic or psychological/psychiatric condition. But also the subgroup of patients with proven mitochondrial disease based upon detection of a pathogenic mutation or an enzyme complex deficiency (group 3, Table 2d) shows a significant correlation between the age-related BMI and the energy-generating capacity. One limiting factor in our study is that proving a primary mitochondrial disorder by elucidating the genetic background is not only time consuming but also not always possible. In many of the patients included, genetic analysis for several, recently recognized nuclear genes are still pending. Strikingly in the "genetically unsolved" subgroup $2(n=80)$ with diminished ATPproduction and respiratory chain enzyme complex deficiencies, four children showed Leigh-like syndrome and two children showed an associated organic aciduria (3-methyl- 
glutaconic aciduria or ethylmalonic aciduria, respectively), symptoms, which are highly suggestive for a primary mitochondrial disorder (Table 3$)$. Concerning the subgroup $4(n=$ 75) with solely diminished ATP-production without respiratory chain enzyme complex deficiencies, one could argue, that a certain percentage of these patients could suffer from a secondary mitochondrial disease. But also this group encompasses patients with multisystem disease $(n=11)$, renal tubular acidosis $(n=1)$, dystonia $(n=1)$, and Leigh-like syndrome $(n=3)$. How can we thus distinguish the primary and secondary cases?

Biochemical analysis in a second tissue could be an important diagnostic tool in primary mitochondrial disease, especially in search for nuclear coded mutations, but as demonstrated in group 2 with proven mutation (Table 1), analysis of the OXPHOS function in fibroblasts was normal in most (23 of 26) cases. Histology was also seldom distinctive. In 172 patients, the (immune) histologic and/or electron microscopic analysis was abnormal only in 25 cases. It is interesting to note that in 20 of these patients a mutation was confirmed, emphasizing the value of histology/electron microscopy in elucidating the underlying genetic defect (Tables 1 and 3).

Children with severe mitochondrial disease are frequently underweight, have eating behavior problems, swallowing difficulties, and frequent infections leading to a decline of their performance and regression of the neurologic picture. Dietary intervention is therefore initialized at an early stage of the disease, mostly by aggressive methods. Significantly, more patients from the group of proven mitochondrial disorders (based on mutation and/or complex deficiency) underwent a tube-feeding before the muscle biopsy compared with the rest of the patients ( 44 of $83=53 \%$ compared with 57 of $172=$ $33 \%$ ) in our study group. In the subgroup of 35 children with severe underweight for age $(\leq-2.5 \mathrm{SD})$, all but one child had a decreased ATP-production; a high percentage of children harbored a mutation $(8 / 35=23 \%)$ or had a deficiency of one or more mitochondrial enzyme complexes $(21 / 35=60 \%)$. Thirteen of these children were treated with tube feeding. Upon our data, that even in the group with proven mitochondrial disease there is still a significant correlation between the age-related BMI and the energy generating capacity, we hypothesize that for these children an aggressive treatment to gain weight by age-appropriate caloric intake will lead to a (partial) improvement in their energy generating capacity. Interestingly, we do not see any correlation between height and ATP-production in the whole group and all investigated subgroups. This supports the hypothesis that the genetic etiology primarily determines the growth in patients with mitochondrial dysfunction. One might speculate that in mitochondrial disease not the age-related height and weight is the aim that should be achieved by nutritional intervention, but the age-appropriate BMI, which is an independent growth parameter.

The origin of all the described problems like failure to thrive, poor weight gain, etc., in children with a mitochondrial dysfunction appears to be multifactorial. For example, the mitochondriopathy can lead to enteral malabsorption, inadequate utilization of absorbed nutrients, central nervous system (CNS) problems leading to a diminished appetite, gastroesophageal reflux or vomiting and decreased activity leading to a worsening of the clinical and biochemical parameters. The energy consumption of a certain patient is also depending on the type of disease, activity, eventual hyperventilation, or seizures. In general, many patients have a higher energy requirement; however, extreme immobility could lead to a decrease in requirements in a later phase of the disease.

Because patients with mitochondrial dysfunction frequently suffer from gastrointestinal problems and eating disorders, optimizing the caloric intake remains a challenge in most cases. One should always consider the influence of nutritional state and growth when interpreting the results of ATPproduction in fresh muscle biopsy. Because of a positive correlation between the age-appropriate BMI and the ATPproduction in muscle, we strongly advise dietary evaluation and intervention, if possible, before performing a muscle biopsy in children with a suspected OXPHOS-disorder. We advise to optimize the nutritional state in children with a suspected mitochondrial disorder before muscle biopsy, if necessary using aggressive methods like tube feeding. In summary, we propose a personalized age-appropriate feeding scheme for each individual patient to optimize the remaining energy-generating capacity.

Acknowledgments. The authors are thankful to the Tjalling Roorda Foundation and to Piet Schouten, Monique van Megen and Maja Klomp-Vos for their support.

\section{REFERENCES}

1. Ushmorov A, Hack V, Dröge W 1999 Differential reconstitution of mitochondrial respiratory chain activity and plasma redox state by cysteine and ornithine in a model of cancer cachexia. Cancer Res 59:3527-3534

2. Morton J, McLoughlin DM, Whiting S, Russell GF 1999 Carnitine levels in patients with skeletal myopathy due to anorexia nervosa before and after refeeding. Int J Eat Disord 26:341-344

3. Chinnery PF, Turnbull DM 1998 Vomiting, anorexia, and mitochondrial DNA disease. Lancet 351:448

4. Sperl W 1997 Diagnosis and therapy of mitochondriopathies. Wien Klin Wochenschr 109:93-99

5. Morava E, Rodenburg R, van Essen HZ, De Vries M, Smeitink J 2006 Dietary intervention and oxidative phosphorylation capacity. J Inherit Metab Dis 29:589

6. Morava E, Dinopoulos A, Kroes HY, Rodenburg RJ, van Bokhoven H, van den Heuvel LP, Smeitink JA 2005 Mitochondrial dysfunction in a patient with Joubert syndrome. Neuropediatrics 36:214-217

7. Estornell E, Tormo JR, Marin P, Renau-Piqueras J, Timoneda J, Barber T 2000 Effects of vitamin A deficiency on mitochondrial function in rat liver and heart. $\mathrm{Br} \mathrm{J}$ Nutr 84:927-934

8. Rafique R, Schapira AH, Coper JM 2004 Mitochondrial respiratory chain dysfunction in ageing; influence of vitamin E deficiency. Free Radic Res 38:157-165

9. Bettendorff L, Sluse F, Goessens G, Wins P, Grisar T 1995 Thiamine deficiencyinduced partial necrosis and mitochondrial uncoupling in neuroblastoma cells are rapidly reversed by addition of thiamine. J Neurochem 65:2178-2184

10. Morava E, van den Heuvel L, Hol F, de Vries MC, Hogeveen M, Rodenburg RJ, Smeitink JA 2006 Mitochondrial disease criteria: diagnostic applications in children. Neurology 67:1823-1826

11. Janssen AJ, Trijbels FJ, Sengers RC, Wintjes LT, Ruitenbeek W, Smeitink JA, Morava E, van Engelen BG, van den Heuvel LP, Rodenburg RJ 2006 Measurement of the energy-generating capacity of human muscle mitochondria: diagnostic procedure and application to human pathology. Clin Chem 52:860-871

12. Janssen AJ, Smeitink JA, van den Heuvel LP 2003 Some practical aspects of providing a diagnostic service for respiratory chain defects. Ann Clin Biochem $40: 3-8$ 